\title{
Predicting Substructure in CDM Haloes
}

\author{
James E. Taylor ${ }^{1} \dagger$, Arif Babul ${ }^{2}$ \\ ${ }^{1}$ Denys Wilkinson Building, 1 Keble Road, Oxford OX1 3RH, United Kingdom \\ email: jet@astro.ox.ac.uk \\ ${ }^{2}$ Elliott Building, 3800 Finnerty Road, Victoria, BC, V8P 1A1, Canada \\ email: babul@uvic.ca
}

\begin{abstract}
Observations of multiple-image gravitational lens systems suggest that the projected mass distributions of galaxy haloes may contain substantial inhomogeneities. The fraction of the halo mass in dense substructure is still highly uncertain, but could be as large as a few percent. While halo substructure is seen in numerical simulations of CDM haloes, little of this substructure survives in the innermost regions of haloes, and thus the observational claims for substructure at small projected radii are slightly surprising. There is evidence, however, that even the highest-resolution simulations published to-date are still limited by numerical effects that heat and disrupt substructure artificially in high-density regions. By comparing numerical and semi-analytic (SA) models of halo substructure, we show that current simulations probably underestimate the mass fraction in substructure at small projected radii, by a factor of at least $2-3$. We discuss the prospects for using lensing observations as a fundamental test of the nature of dark matter.
\end{abstract}

\section{Introduction: The case for substructure in observed haloes}

There is now overwhelming evidence that the matter content of the Universe is in large part non-baryonic (Spergel et al. 2003). The strongest contender for this extra component is some sort of weakly interacting cold dark matter (CDM), possibly in the form of a relic supersymmetric particle. One of the most surprising properties of CDM is its tendency to cluster gravitationally on subgalactic (and possibly even possibly subsolar) scales. Detecting subgalactic lumps of dark matter would be a dramatic confirmation of CDM theory, and should be considered a top priority for observational astrophysics.

Strong lensing has long been recognised as a method for identifying dark, compact objects on subgalactic scales (e.g. Press \& Gunn 1973; Blandford \& Jaroszynski 1981; Wambsganss \& Paczynski 1992). Interest in these tests was recently revived by Mao \& Schneider (1998), who proposed that some form of substructure - either a normal baryonic feature such as a spiral arm or a globular cluster, or substructure within the dark matter halo of the system - might explain why the flux ratios of the different components of the multiple-image system B1422+231 disagreed so strongly with the predictions of models assuming smooth lens potentials.

The idea that anomalous flux ratios might be due to substructure was reexamined more recently by several authors (Metcalf \& Madau 2001; Chiba 2002; Dalal \& Kochanek 2002). Since these initial papers, there has been extensive work trying to extract more quantitative information from the observations (e.g. Metcalf \& Zhao 2002; Metcalf 2002; Bradac et al. 2002; Schechter \& Wambsganss 2002; Chen, Kravtsov \& Keeton 2003; Keeton, Gaudi \& Peters 2003), but there is increasing concern that other phenomena such as microlensing may be responsible for the deviations observed (Schechter \& Wambsganss 2002), or that they may simply be due to insufficiently general modelling of the lens 
potential (Evans \& Witt 2003). Currently there still seems good evidence for genuine anomalies in a few systems (Moustakas \& Metcalf 2003; Metcalf et al. 2004; Metcalf these proceedings), suggesting substructure in the projected mass distribution at the level of a few percent, but continued observations at many different wavelengths will be required to disentangle the effects of substructure from microlensing, scintillation or other phenomena. The observational effort is worthwhile, however, as it may provide the first hard evidence to justify one of the main assumptions of our current cosmological model, the cold, collisionless nature of dark matter.

\section{A new approach to modelling CDM haloes}

Whatever the status of the substructure problem observationally, it is not clear that there is a robust theoretical prediction with which to compare the lensing results. Dark matter haloes form through the gravitational collapse of diffuse dark matter, as well as the hierarchical merging of smaller haloes. The process is sufficiently non-linear that most of our understanding of it comes from numerical simulations. The strong lensing anomalies depend on the net mass fraction in relatively low-mass substructure $\left(10^{5} M_{\odot}-10^{7} M_{\odot}\right)$, projected on the central few kiloparsecs of galaxy haloes. This is close to, or beyond, the formal resolution limit of most current simulations, and even in those simulations that can resolve structures on this scale, serious doubts remain as to the completeness of the results in the innermost parts of the halo. Thus, while simulations currently predict less central substructure than inferred from observations, this may be partly due to their limited resolution.

To study halo substructure on smaller scales or very close to the centre of the halo, we have developed an alternative, semi-analytic model (Taylor \& Babul 2004; Taylor \& Babul in preparation). This model includes several distinct components. First, merger histories for a large number of individual haloes are generated randomly, using Press-Schechter statistics and the merger-tree algorithm of Somerville and Kolatt (1999), together with a correction for higher-order substructure developed in Taylor \& Babul (2004). Each merging subhalo is then placed on a random orbit starting at the virial radius of the main system, and evolved using the analytic model of satellite dynamics described in Taylor \& Babul (2001), experiencing orbital decay due to dynamical friction, and heating and stripping due to tidal forces. The properties of the main system change dynamically over time, and the formation of a galaxy can also be modelled schematically, as described in Taylor \& Babul (2003), further modifying the central mass distribution.

Overall, this model provides a computationally efficient way of simulating the hierarchical assembly of galaxy or cluster haloes, and the evolution of their substructure. Because it performs only a few calculations per lump of dark matter (or 'subhalo') merging with the main system it can be used to track the evolution of many thousands of subhaloes in a typical system, providing complete information about halo substructure down to masses around $10^{5}-10^{6} M_{\odot}$.

\section{Results: The outer halo}

To test the accuracy of the SA model, we have used it to generate a large set of galaxy haloes and compared their substructure to the substructure found in a set of high-resolution simulations of halo formation by Ghigna et al. (1998, 2000) and Moore et al. (1999a, 1999b). These include the galaxy-mass haloes 'Andromeda' and the 'Milky Way' (the 'Local Group' - Moore et al. 1999b) and cluster-mass haloes 'Coma', 'Virgo I' and 'Virgo II' (Virgo IIa and b are two different outputs from the same simulation). 

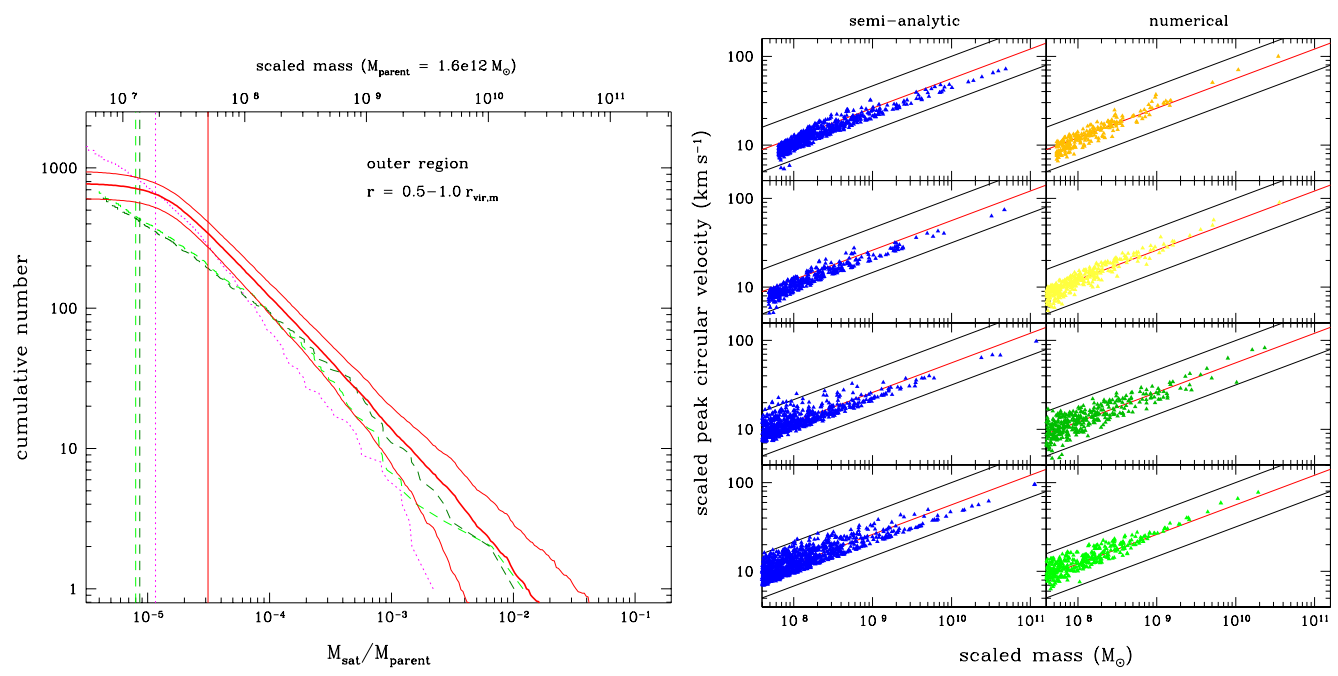

Figure 1. (Left) The cumulative mass function predicted by the SA model in the outer parts of the halo. The thick lines show the average result for a hundred SCDM merger trees at $z=0$. The thin solid lines show the 1- $\sigma$ variance for this set. The dashed and dotted lines are the normalised cumulative mass functions measured in three high-resolution simulations (dashed lines - Virgo IIa and IIb; dotted lines - Coma). The vertical lines indicate the resolution limit of the SA trees (solid) and the 32-particle mass limits of the simulations (dotted and dashed). (Right) The distribution of subhaloes as a function of their mass and of their peak velocity, in the SA model (left-hand plots) and the simulations (right-hand plots; values are scaled to the SA halo mass and velocity).

Each was resolved with $\sim 10^{6}$ particles of more, and with a softening length of less than $1 \%$ of the virial radius.

The left-hand plot in figure 1 compares the cumulative distribution of subhalo masses in the outer regions of the SA haloes (thick solid line) with the simulations (dashed or dotted lines). The masses have been scaled to the mass of the parent halo in the SA model, $1.6 \times 10^{12} M_{\odot}$, for comparison. Overall we find an excellent match in the outer regions of the halo. The simulations have an average amplitude about $20 \%$ lower than the SA average, but this is only 1-2 times the halo-to-halo scatter (thin solid lines). We note that this agreement is achieved without adjusting any free parameters - the parameters in the semi-analytic model have all been fixed previously by other considerations, as discussed in paper Taylor \& Babul (2004).

The right-hand plot in figure 1 shows that the internal structure of subhaloes is also very similar. The peak circular velocity of each system is plotted versus its mass, for the SA models (left-hand plots) and the numerical models (right-hand plots). Values are scaled as in figure 1. The effects of softening and shot noise in the number of particles within the peak radius of each subhalo have been added to SA results, since these effects are present in the numerical data. With this correction, we find very good agreement between the distributions of subhalo properties.

\section{Results: The inner halo}

In the inner halo, on the other hand, the SA model run with the same parameters as above predicts 2-3 times more substructure than is seen in the numerical simulations. Moreover, the distribution of substructure is much more centrally concentrated than in the simulations. Figure 2 compares the radial distribution of subhaloes in the SA models 


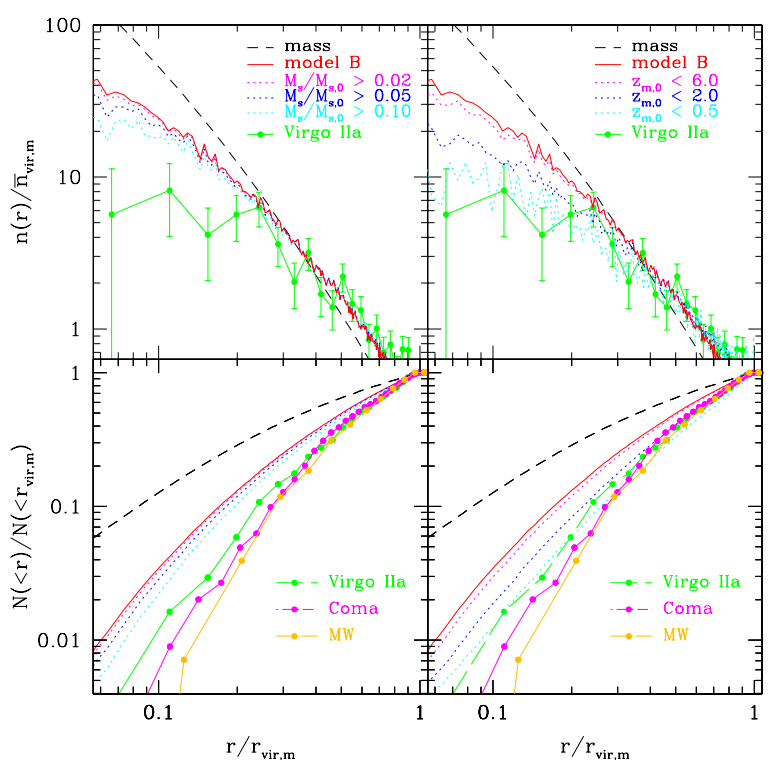

Figure 2. Top panels: The number density of subhaloes in the SA haloes (upper solid lines) and in three simulations (connected points with error bars). To avoid incompleteness, the results are cut at the equivalent of $5 \times 10^{7} M_{\odot}$. In each case the density is relative to the mean within the virial radius. The dashed line shows the density profile of the main halo, normalised to the mean within the virial radius. Bottom panels: The cumulative number of subhaloes vs. radius, normalised to the number within the virial radius, for the same mass cuts as in the top panel. The dashed lines show the mass of the main halo interior to a given radius, normalised to the mass within the virial radius. The dotted lines in the left-hand panels show the results of ignoring highly stripped systems; the dotted lines in the right-hand panels show the results of ignoring old systems.

(solid and dotted lines) and three simulations (dashed or dot-dashed lines + data points). The smooth dashed curve shows the overall mass distribution of the parent halo. While both SA and numerical subhaloes are antibiased with respect to the background mass distribution, the SA model predicts a central density of substructure 3-4 times higher than the numerical value. Removing highly-stripped systems (left-hand panel) does not affect this result very strongly, but removing old systems (right-hand panel) does. Thus the SA model predicts the existence of an extra population of old systems, deep in the centre of the halo.

The importance of these results for lensing detections of halo substructure is illustrated in figure 3. This compares the projected mass function within various radii around a galaxy halo (top panels), and the fraction of the projected surface density in substructure (bottom panels), for the SA model and the highest-resolution simulation (solid and dashed lines respectively). The offset of $\sim 2$ between the two, when averaged over large projected radii (leftmost panel), grows to roughly an order of magnitude at small projected radii (3rd and 4th panels).

\section{Summary and future prospects}

When observations can reliably determine the clustering of dark matter on subgalactic scales, they will shed light on a number of important questions in fundamental physics. The nature and amplitude of small-scale CDM structure depends on the spectrum of perturbations generated towards the end of inflation, which in turn depends on the 


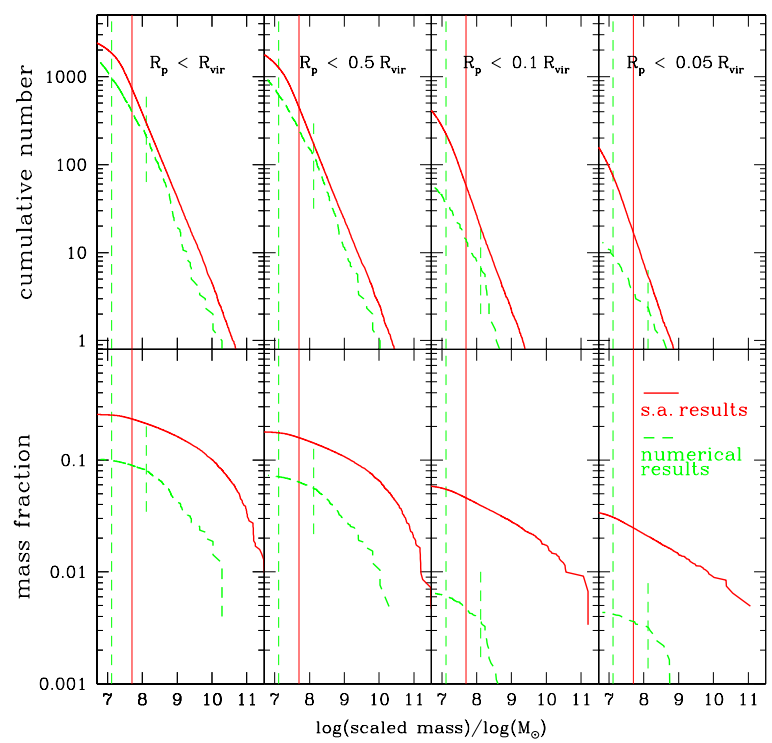

Figure 3. (Top panel) Cumulative mass functions for subhaloes within some projected radius $R_{\mathrm{p}}$, for the SA model (solid lines) and Virgo IIa (dashed lines). (Bottom Panel) The fraction of the projected mass within $R_{\mathrm{p}}$ contained in subhaloes of mass $M$ or larger. The numerical results are the average over three different projections. Vertical lines indicate the resolution limit of the merger tree (solid) and the 32-particle mass limits of the simulation (dashed).

primordial spectral index, the shape of inflationary potential, and the physics of reheating. The subsequent growth of these perturbations also depends on the equation of state of the universe at the quark-hadron transition and at nucleosynthesis. Finally, the evolution of small-scale structure at late times is a sensitive test of dark matter physics, such as interactions or annihilation. These small-scale properties will also have an important effect on the growth of visible structure, especially at very high redshift during the epoch of reionization.

To tap the potential of recently developed methods for detecting substructure in strongly lensed systems, we need robust predictions for the behaviour of dark matter on very small scales, at very high densities, over cosmological timescales. Achieving this goal remains a challenge for current numerical simulations of structure formation. We have presented initial results from a semi-analytic model which uses halo merger trees and satellite dynamics to model the properties of substructure within dark matter haloes. Without any adjustment of free parameters, this model matches the results of highresolution simulations very closely in the outer parts of haloes, where the simulations are most likely to be accurate. In the inner parts, however, it predicts central densities of substructure 3-4 times higher than those found in simulations. This may help explain the very high levels of substructure inferred from recent lensing observations.

\section{Acknowledgements}

The authors wish to thank E. Hayashi, S. Ghigna, B. Moore, J. Navarro and T. Quinn for providing the substructure data from their simulations. JET gratefully acknowledges support from PPARC UK. AB gratefully acknowledges support from NSERC Canada, through the Discovery and the Collaborative Research Opportunities (CRO) programs. 


\section{References}

Blandford, R. D. \& Jaroszynski, M. 1981, ApJ 246, 1.

Bradač, M., Schneider, P., Steinmetz, M., Lombardi, M., King, L. J., \& Porcas, R. 2002, A\& $A p$ $388,373$.

Chiba, M. 2002, ApJ 565, 17.

Chen, J., Kravtsov, A. V., \& Keeton, C. R. 2003, ApJ 592, 24.

Dalal, N. \& Kochanek, C. S. 2002, ApJ 572, 25.

Eke, V. R., Navarro, J. F., \& Steinmetz, M. 2001, ApJ 554, 114.

Evans, N. W. \& Witt, H. J. 2003, MNRAS 345, 1351.

Ghigna, S., Moore, B., Governato, F., Lake, G., Quinn, T., \& Stadel, J. 1998, MNRAS 300, 146.

Ghigna, S., Moore, B., Governato, F., Lake, G., Quinn, T., \& Stadel, J. 2000, ApJ 544, 616.

Keeton, C. R., Gaudi, B. S., \& Petters, A. O. 2003, ApJ 598, 138.

Mao, S. \& Schneider, P. 1998, MNRAS 295, 587.

Metcalf, R. B. 2002, ApJ 580, 696.

Metcalf, R. B. 2004, ApJ submitted (astro-ph/0407298).

Metcalf, R. B. \& Madau, P. 2001, ApJ 563, 9.

Metcalf, R. B. \& Zhao, H. 2002, ApJL 567, L5.

Metcalf, R. B., Moustakas, L. A., Bunker, A. J., \& Parry, I. R. 2004, ApJ 607, 43.

Moore B., Ghigna S., Governato F., Lake G., Quinn T., Stadel J., \& Tozzi P. 1999, ApJ 524, L19.

Moore, B., Quinn, T., Governato, F., Stadel, J., \& Lake, G. 1999, MNRAS 310, 1147.

Moustakas, L. A. \& Metcalf, R. B. 2003, MNRAS 339, 607.

Press, W. H. \& Gunn, J. E. 1973, ApJ 185, 397.

Schechter, P. L. \& Wambsganss, J. 2002, ApJ 580, 685.

Somerville R. S. \& Kolatt T. S. 1999, MNRAS 305, 1.

Spergel, D. N. et al. 2003, ApJS 148, 175.

Taylor, J. E. \& Babul, A. 2001, ApJ 559, 716.

Taylor, J. E. \& Babul, A. 2003, Ap\&SS 284, 405.

Taylor, J. E. \& Babul, A. 2004, MNRAS 348, 811.

Wambsganss, J. \& Paczynski, B. 1992, ApJL 397, L1.

\section{Discussion}

L. KING: If you take your predicted substructure into account, will it explain the observations?

J. TAYLOR: Yes, or at least it almost will. The status of the observations is a bit unclear, but in the few systems were we do have strong evidence for substructure, the inferred level is high - within a few percent of the virial radius, a few percent of the projected mass density appears to be in substructure. This is at least ten times more than the simulations predict, but would be close to or just above the level the SA model predicts. The SA model also predicts a factor of 3 variation from one system to the next, however, so we really need to observe more systems. 